\title{
UM RETRATO DA ENGENHARIA DE PRODUÇÃO NO BRASIL
}

\section{AN ANALYSIS OF THE INDUSTRIAL ENGINEERING IN BRAZIL}

\author{
Cristhiano Stefani Faé ${ }^{1}$ José Luis Duarte Ribeiro ${ }^{2}$ \\ ${ }^{1}$ Mestrando, Laboratório de Otimização de Produtos e Processos - LOPP, Programa de Pós- \\ Graduação em Engenharia de Produção - PPGEP, fae@producao.ufrgs.br \\ ${ }^{2}$ Doutor, Universidade Federal do Rio Grande do Sul - UFRGS, Praça Argentina 9, ${ }^{\circ}$ andar, \\ sala LOPP, Centro, Porto Alegre-RS/Brasil, CEP 90.040-020, ribeiro@producao.ufrgs.br
}

Recebido para publicação em: 10/03/2005
Aceito para publicação em: $15 / 07 / 2005$

\section{Resumo}

A Engenharia de Produção tem demonstrado um crescimento notório nos últimos anos no Brasil. Este artigo apresenta um estudo quantitativo da evolução e localização dos cursos de graduação em Engenharia de Produção no país. Foram levados em conta aspectos da evolução do número de vagas e instituições de ensino que oferecem o curso, bem como a relação da oferta de vagas com fatores econômicos e sociais das regiões onde estes cursos estão instalados. Os resultados deste estudo revelam que a oferta de cursos nas regiões Centro-Oeste e Nordeste é relativamente pequena, tendo em vista, respectivamente, o PIB e a população dessas regiões.

Palavras-chave: Engenharia de Produção, Instituições de ensino, Graduação

\section{Introdução}

A primeira instituição de ensino a oferecer o curso de Engenharia de Produção no Brasil foi a Escola Politécnica da Universidade de São Paulo, no ano de 1957, sob a coordenação do Prof. Ruy Aguiar da Silva Leme. Uma década após, seguindo esse mesmo exemplo, a FEI - Faculdade de Engenharia Industrial de São Bernardo do Campo abriu o seu curso em 1967.

De lá para cá, o crescimento dos cursos de Engenharia de Produção no Brasil tem sido notável. A origem desse crescimento, provavelmente, são os desafios e necessidades atuais do mundo empresarial. Segundo Cunha (2001), as origens históricas da engenharia remontam à época da Revolução Industrial, onde o foco da demanda torna-se o foco da solução de problemas. Naquele momento, surgiram os cursos de diversos ramos da Engenharia, na expectativa de prover o conhecimento necessário à produção em larga escala. Todavia, ao passar dos anos, a questão-chave 
tornou-se, além de produzir cada vez mais e melhor, otimizar o uso de recursos e materiais. É nesse contexto, em meados do século XX, que a Engenharia de Produção encontra suas raízes históricas, pela sua grande capacidade de formar profissionais habilitados a solucionar problemas e, acima de tudo, gerenciar sistemas produtivos.

Segundo Leme (1983), a prática da Engenharia de Produção é bastante antiga, com os primeiros indícios encontrados na Inglaterra na época da Revolução Industrial. Porém, o nascimento da Engenharia de Produção, como é geralmente aceito, se deu nos Estados Unidos, no período de 1882 a 1912, com o surgimento e desenvolvimento do denominado "Scientific Management", obra de um grupo de engenheiros: F.W. Taylor, Frank e Lillian Gilbreth, H.L. Gantt, H. Emerson, entre outros. Leme afirma que, apesar de muito atacado e controvertido, o Scientific Management passou a ser introduzido em inúmeras empresas por consultores que se intitulavam "Industrial Engineers". Foi neste momento que surgiu a Industrial Engineering, o nome pelo qual a Engenharia de Produção é conhecida nos Estados Unidos.

No Brasil, a criação dos cursos de Engenharia de Produção foi impulsionada pela forte mudança no mercado de trabalho provocada pela instalação de diversas multinacionais no país na década de 50. Conforme explica Leme (1983), estas empresas, especialmente as Norte-Americanas, possuíam nos seus organogramas posições que nas matrizes eram ocupadas por "Industrial Engineers", como, por exemplo, os departamentos de tempos e métodos, de planejamento e controle de produção, de controle de qualidade, entre outros.

No cenário atual, de acirrada competitividade, integração entre os mercados globais, demanda por produtos de alta qualidade e empresas cada vez mais "enxutas", é visível a necessidade de recursos humanos compatíveis com tais atribuições e desafios de gestão. Neste sentido, o Engenheiro de Produção vem tornando-se uma peça fundamental para as empresas, quer sejam dos ramos da indústria, comércio ou serviços. No Brasil, a realidade não é diferente e, de forma crescente, as instituições de ensino passam a oferecer o curso de Engenharia de Produção.

Este artigo tem o objetivo de apresentar um estudo da oferta e localização dos cursos de graduação em Engenharia de Produção (EP) no Brasil, considerando a situação econômica das regiões onde estes cursos estão instalados. O estudo envolve o mapeamento, realizado em 2004, de todas as instituições de ensino que possuem estes cursos em funcionamento, ofertados nos diversos estados do Brasil. A partir daí é realizada uma comparação da oferta de cursos e vagas com os indicadores econômicos e sociais de cada região. A seção 2 apresenta um panorama da evolução das escolas de EP no Brasil. A seção 3 descreve a metodologia utilizada na pesquisa. A seção 4 apresenta os dados coletados em 2004, permitindo associar o número de cursos de EP com a população e o PIB das regiões onde eles estão inseridos. A seção 5 discute os dados apresentados, indicando as regiões e estados onde existe deficiências na oferta de formação de profissionais de 
EP. Finalizando, a seção 6 apresenta as conclusões do trabalho.

\section{Evolução da Engenharia de Produção no Brasil}

Ao longo dos últimos anos, os cursos de Engenharia de Produção no Brasil vem apresentando um crescimento acentuado. Diversos cursos estão sendo criados, tanto em nível de graduação, como de pós-graduação. Além disso, há um grande movimento de mudança nas ênfases dadas nos cursos já existentes: aqueles que até então apresentavam uma habilitação específica estão rumando para a chamada Engenharia de Produção "plena".

Em âmbito empresarial, a evolução do curso também é crescente. Observa-se que rapidamente se desfaz a confusão original referente ao entendimento do que é a Engenharia de Produção e o papel do Engenheiro de Produção. Um exemplo disso pode ser verificado neste trecho extraído da Revista Veja: "No caso do engenheiro de produção, especialidade das mais valorizadas na praça, ele elabora e desenvolve projetos que aumentem a produção numa fábrica, mas reduzam os custos. É a discussão do momento em todas as empresas. Seu passe é disputado por bancos, empresas de consultoria e indústrias” (Revista Veja - Edição 1805 - 04 de junho de 2003, página 76).

O quadro 1 deixa clara a grande expansão no número de escolas que oferecem o curso de graduação em Engenharia de Produção. Conforme pode ser visto, a oferta de cursos vem apresentando um crescimento exponencial. Para comprovar esta afirmativa, basta comparar na tabela os dados do ano de 1998 com os de 2002, onde foi constatado um aumento em mais de 50\% no número de cursos no Brasil. Esse crescimento poderia ser justificado pela maior aceitação do Engenheiro de Produção formado por parte das empresas, bem como pelo maior conhecimento do que é esta modalidade de Engenharia.

No Brasil, são encontrados dois tipos de cursos de Engenharia de Produção. Existem os cursos ditos plenos e os cursos que funcionam com habilitações específicas (ênfases) de um dos ramos tradicionais da Engenharia. No quadro 2, verifica-se um grande número de cursos de Engenharia de Produção com habilitação específica em outros ramos da Engenharia, bem como a diversidade destas habilitações. Por outro lado, o curso pleno é responsável por quase $50 \%$ de todos os cursos existentes, apresentando 51 cursos em relação a outros 59 com ênfases. Vale salientar também o surgimento de novas habilitações nos cursos de Engenharia de Produção, impulsionadas pela atual demanda do mercado. Como exemplo, podemos citar os cursos com ênfase em tecnologias limpas, software e calçados e componentes. 
QUADRO 1 - Evolução dos cursos no Brasil

\begin{tabular}{|c|c|}
\hline ANO & $\mathbf{N}^{\mathbf{0}}$ CURSOS \\
\hline 1957 & 1 \\
\hline 1967 & 2 \\
\hline 1993 & 17 \\
\hline 1996 & 20 \\
\hline 1998 & 35 \\
\hline 2002 & 76 \\
\hline 2004 & 110 \\
\hline
\end{tabular}

Fonte: Adaptado da Revista Pesquisa e Tecnologia FEI, 2002.

QUADRO 2 - Cursos de EP no Brasil subdivididos por ênfase

\begin{tabular}{|c|c|}
\hline CURSOS & TOTAL \\
\hline Produção & 51 \\
\hline Produção Mecânica & 21 \\
\hline Produção Civil & 11 \\
\hline Produção Elétrica & 8 \\
\hline Produção Agroindustrial & 7 \\
\hline Produção Química & 4 \\
\hline Produção Metalúrgica & 3 \\
\hline Produção Materiais & 1 \\
\hline Produção Têxtil & 1 \\
\hline Produção Calçados e Componentes & 1 \\
\hline Produção: Tecnologias + Limpas & 1 \\
\hline Produção Software & 1 \\
\hline Total & $\mathbf{1 1 0}$ \\
\hline
\end{tabular}

Fonte: Adaptado da Revista Pesquisa e Tecnologia FEI, 2002.

Segundo Cunha (2002), os cursos do tipo pleno concentram quase toda a sua carga horária profissionalizante no estudo da gestão da produção, enquanto que os de habilitação específica dividem essa carga entre esse estudo e o dos sistemas técnicos - normalmente, priorizando este último por larga margem. Deve-se notar que a legislação atualmente em vigor considera apenas os egressos do primeiro tipo de curso como Engenheiros de Produção. A figura 1 mostra o relacionamento entre as áreas de conhecimento supracitadas.

\begin{tabular}{|c|c|c|c|}
\hline \multirow{7}{*}{$\begin{array}{c}\text { Ciências da Administração } \\
\text { de empresas }\end{array}$} & \multirow{7}{*}{$\begin{array}{l}\text { Engenharia } \\
\text { de Produção }\end{array}$} & Engenharia de Produção Civil & Engenharia Civil \\
\hline & & Engenharia de Produção Mecânica & Engenharia Mecânica \\
\hline & & Engenharia de Produção Química & Engenharia Química \\
\hline & & Engenharia de Produção Elétrica & Engenharia Elétrica \\
\hline & & Engenharia de Prod. Metalúrgica & Engenharia Metalúrgica \\
\hline & & Engenharia de Produção Mecânica & Engenharia Química \\
\hline & & & Engenharia de Minas \\
\hline
\end{tabular}

\section{Formação Teórica Predominantemente Gerencial}

Formação Teórica Predominantemente Tecnicista

Fonte: Cunha (2002).

Figura 1 - Relação da Engenharia de Produção com as demais áreas da Engenharia 
Cunha (2002) ressalta que o aparecimento da Engenharia de Produção com uma componente mais gerencial deveu-se, provavelmente, ao fato dos cursos da área das Ciências da Administração de Empresas conduzirem seus egressos a uma formação de característica mais analítica, sem o foco principal na resolução de problemas, característica bem mais típica da Engenharia. Esta diferenciação torna o profissional de engenharia de produção apto a lidar com problemas relacionados com a mobilização de recursos técnicos, dentro da função de cumprir as tarefas a que se destina a empresa ou instituição a que serve.

\section{Metodologia}

A abordagem adotada segue a linha de uma pesquisa virtual, utilizando informações disponíveis na Internet somadas às informações das instituições cadastradas na ABEPRO Associação Brasileira de Engenharia de Produção e informações econômicas e sociais encontradas no Instituto Brasileiro de Geografia e Estatística (IBGE).

Os dados reunidos foram tabulados e processados com a utilização do software Excel, onde foram feitos os estudos estatísticos. Primeiramente, procurou-se fazer um mapeamento de todos os cursos de graduação em Engenharia de Produção existentes nas instituições de ensino presentes no Cadastro Nacional da ABEPRO. Após a consolidação destas informações, partiu-se para, em um segundo momento, fazer a totalização dos cursos subdividindo-os pelos estados e regiões a que pertenciam. O resultado deste cálculo permitiu a elaboração de um ranking de ocorrência. Por fim, realizou-se uma análise estatística comparando as informações dos cursos de Engenharia de Produção e do número de vagas ofertadas nestes cursos com os dados de PIB (Produto Interno Bruto) e População. Para fins de análise, ambos os dados foram agrupados nas cinco regiões do Brasil.

Vale salientar que este material faz parte de uma ampla pesquisa do perfil do profissional graduado em Engenharia de Produção, projeto idealizado pela ABEPRO.

\section{Análise dos dados}

O quadro 3 apresenta um resumo de todas as informações utilizadas no estudo. Conforme pode ser verificado, o estado São Paulo, pioneiro na implantação da primeira escola de Engenharia de Produção, é o estado que possui o maior número de cursos e número de vagas ofertadas, assim como os maiores valores de PIB e população. Na seqüência aparecem os estados do Rio de Janeiro, Minas Gerais, Rio Grande do Sul e demais. 
QUADRO 3 - Cursos de EP, vagas ofertadas, PIB e População dos estados brasileiros

\begin{tabular}{ccccc}
\hline Estados & $\mathbf{N}^{\mathbf{0}}$ cursos & Vagas & PIB x 10^6 & População x 10^6 \\
\hline SP & 30 & 2950 & 370,8 & 37,03 \\
RJ & 17 & 1010 & 137,9 & 14,39 \\
RS & 10 & 770 & 106,2 & 10,19 \\
MG & 9 & 480 & 85,1 & 17,89 \\
PR & 9 & 340 & 42,4 & 9,56 \\
SC & 8 & 605 & 66,0 & 5,36 \\
BA & 6 & 460 & 48,2 & 13,07 \\
ES & 5 & 430 & 21,5 & 3,10 \\
AM & 3 & 240 & 18,9 & 2,81 \\
CE & 3 & 172 & 20,8 & 7,43 \\
PE & 2 & 260 & 29,1 & 7,92 \\
AL & 1 & 60 & 29,6 & 2,82 \\
DF & 1 & 120 & 21,7 & 2,05 \\
GO & 1 & 100 & 18,9 & 5,00 \\
MT & 1 & 50 & 13,4 & 2,50 \\
PA & 1 & 50 & 9,3 & 6,19 \\
PB & 1 & 40 & 9,2 & 3,44 \\
RN & 1 & 45 & 7,0 & 2,78 \\
SE & 1 & 120 & 5,9 & 1,78 \\
\hline TOTAL & $\mathbf{1 1 0}$ & $\mathbf{8 3 0 2}$ & $\mathbf{1 0 6 2 , 0}$ & \\
\hline
\end{tabular}

Cabe salientar que algumas instituições apresentaram mais de uma opção de curso, ou seja, mantinham, por exemplo, um curso de Engenharia de Produção com habilitação em Mecânica, outro em Civil e outro em Elétrica. Para fins de análise, essas instituições, bem como o estado a que pertenciam receberam peso três (3) no somatório de ocorrências. Outro aspecto importante é o fato de que apenas 19 estados brasileiros apresentavam alguma instituição de ensino detendo o curso na sua grade de ofertas.

Para apoiar o estudo foram realizados gráficos de correlação, cruzando o número de cursos e vagas ofertadas com o PIB e a população das regiões do Brasil.

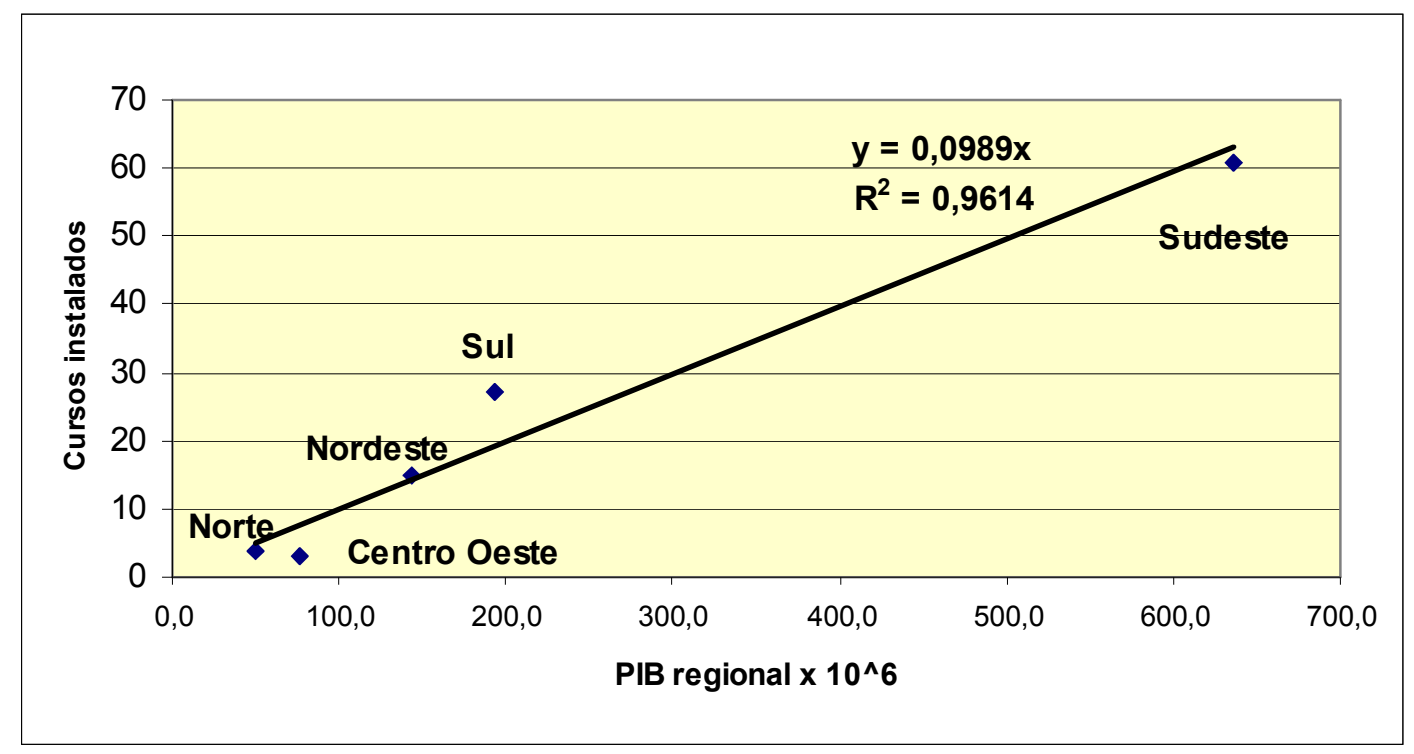

Figura 2 - Número de cursos em função do PIB regional 


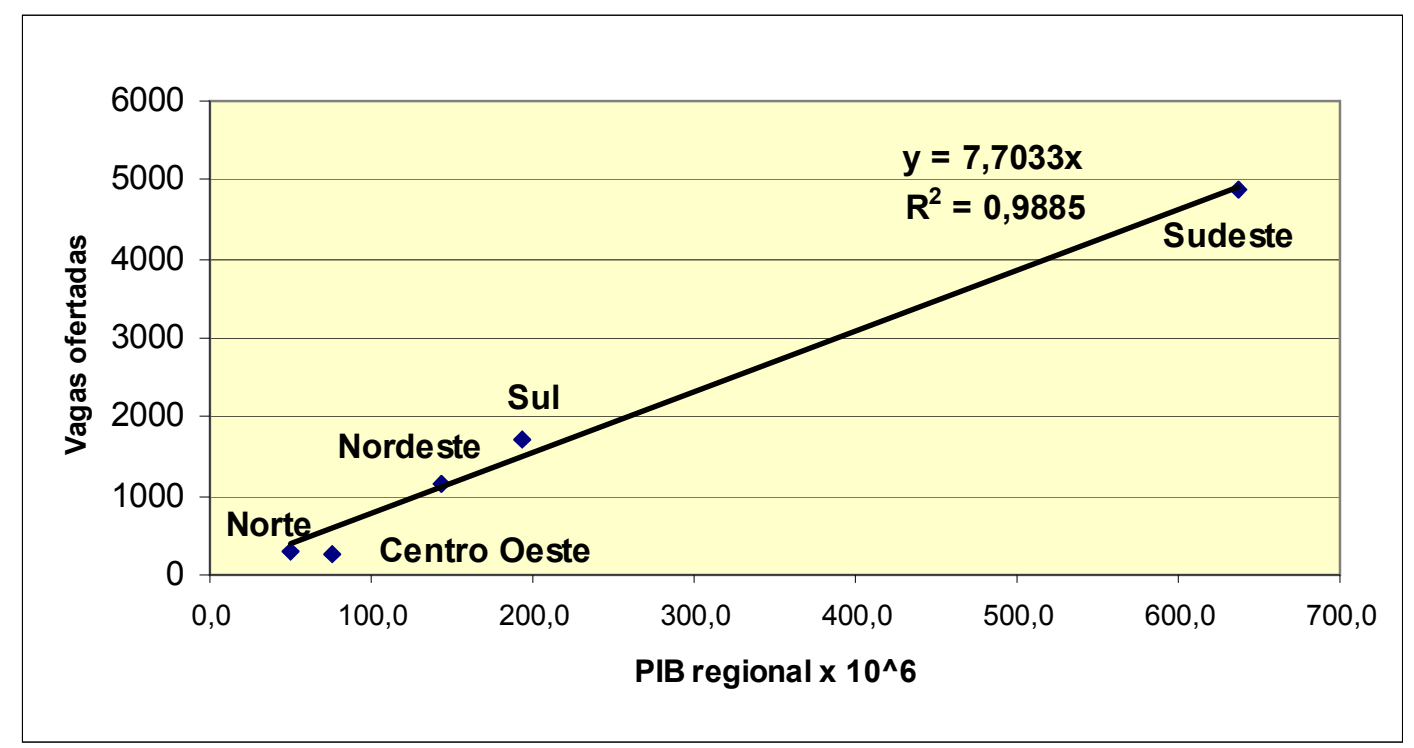

Figura 3 - Oferta de vagas em função do PIB regional

O estudo de correlação que levou em conta os valores dos PIBs regionais está apresentado nas figuras 2 e 3. A região Sudeste possui o maior número de cursos e também o maior PIB regional do país, são sessenta (60) cursos de graduação em EP com um PIB de R\$ 636,395 milhões. Na seqüência, encontramos a região Sul com um PIB de R\$193,535 milhões, contando com 22 cursos. Em terceiro lugar, está a região Nordeste, que apresenta um PIB de R\$ 144,136 milhões e 15 cursos ofertados. A região Norte vem em seguida com 5 cursos e um Produto Interno de R\$50,650 milhões. Por fim, encontra-se a região Centro-Oeste, que possui 3 cursos e um PIB de 76,541 milhões de reais.

Considerando os dados apresentados nas figuras 2 e 3, é possível observar que o comportamento dos pontos segue, aproximadamente, uma linha reta. Ou seja, há uma relação direta entre o número de cursos ofertados e o PIB. Verifica-se a grande distância numérica da região Sudeste, considerando o número de cursos oferecidos e o do PIB regional. Sucessivamente aparecem as regiões Sul e Nordeste, seguidas das regiões Centro-Oeste e Norte.

Pode-se perceber que a região Centro-Oeste, apesar de possuir um PIB mais elevado que a região Norte, possui a menor oferta de vagas de graduação. Essa oferta de vagas é claramente insuficiente, principalmente considerando que a vertente agro-industrial, principal componente do PIB da região Centro-Oeste, é uma importante modalidade que pode ser desenvolvida no âmbito dos cursos de Engenharia de Produção.

A análise estatística comprova a consistência da relação linear entre Número de cursos e PIB, uma vez que o coeficiente R2, que avalia a adequação do ajuste dos modelos lineares foi, respectivamente, 0,961 (96,1\% da variabilidade observada na oferta de cursos é explicada pelo PIB regional) e 0,988 (98,8\% da variabilidade observada na oferta de vagas é explicada pelo PIB regional). 
Outra análise relevante para o trabalho, refere-se ao estudo de correlação entre a oferta de cursos ou vagas e a população regional. Este estudo aparece sumarizado nas figuras 4 e 5 . Nesta etapa, foram considerados os dados da população brasileira, também divididos por regiões do país, e a seguir foi feita a comparação com o número de cursos e respectiva oferta de vagas.

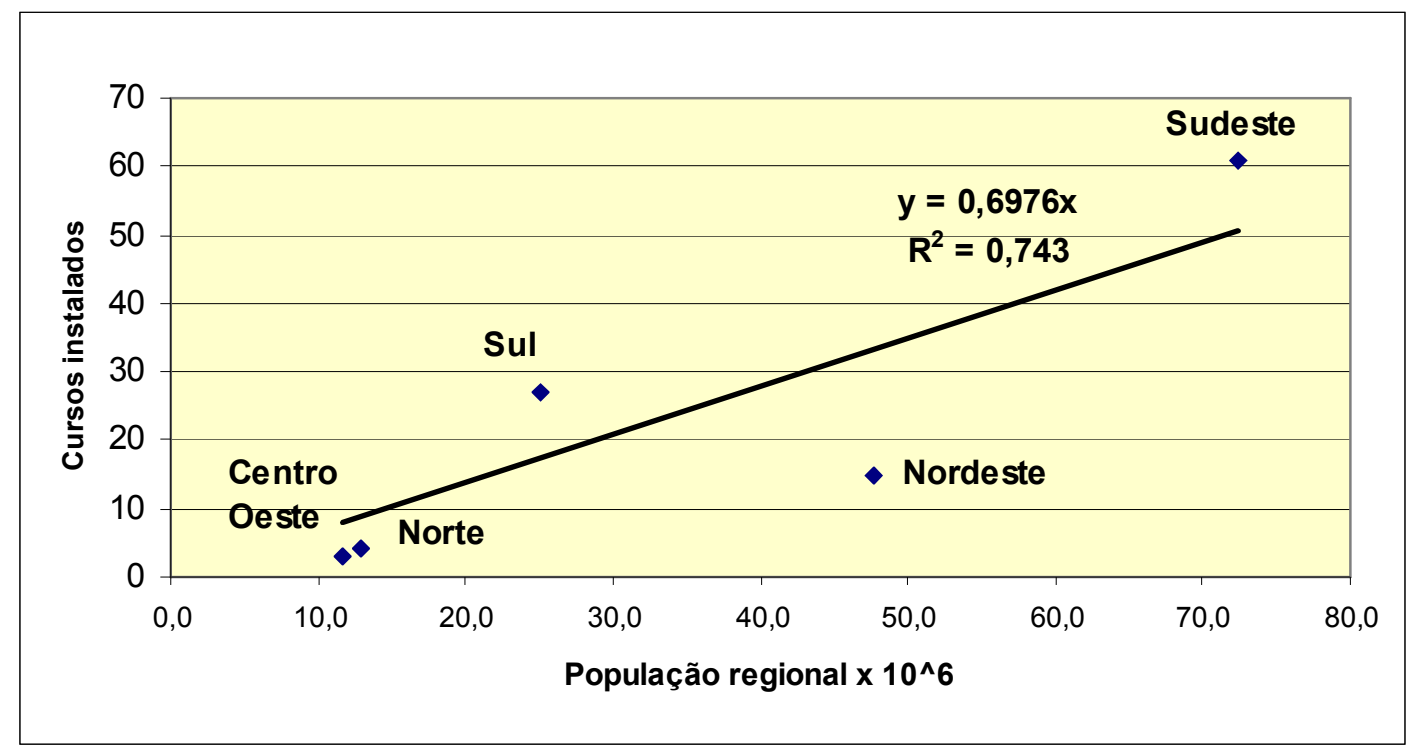

Figura 4 - Número de cursos em função da população regional

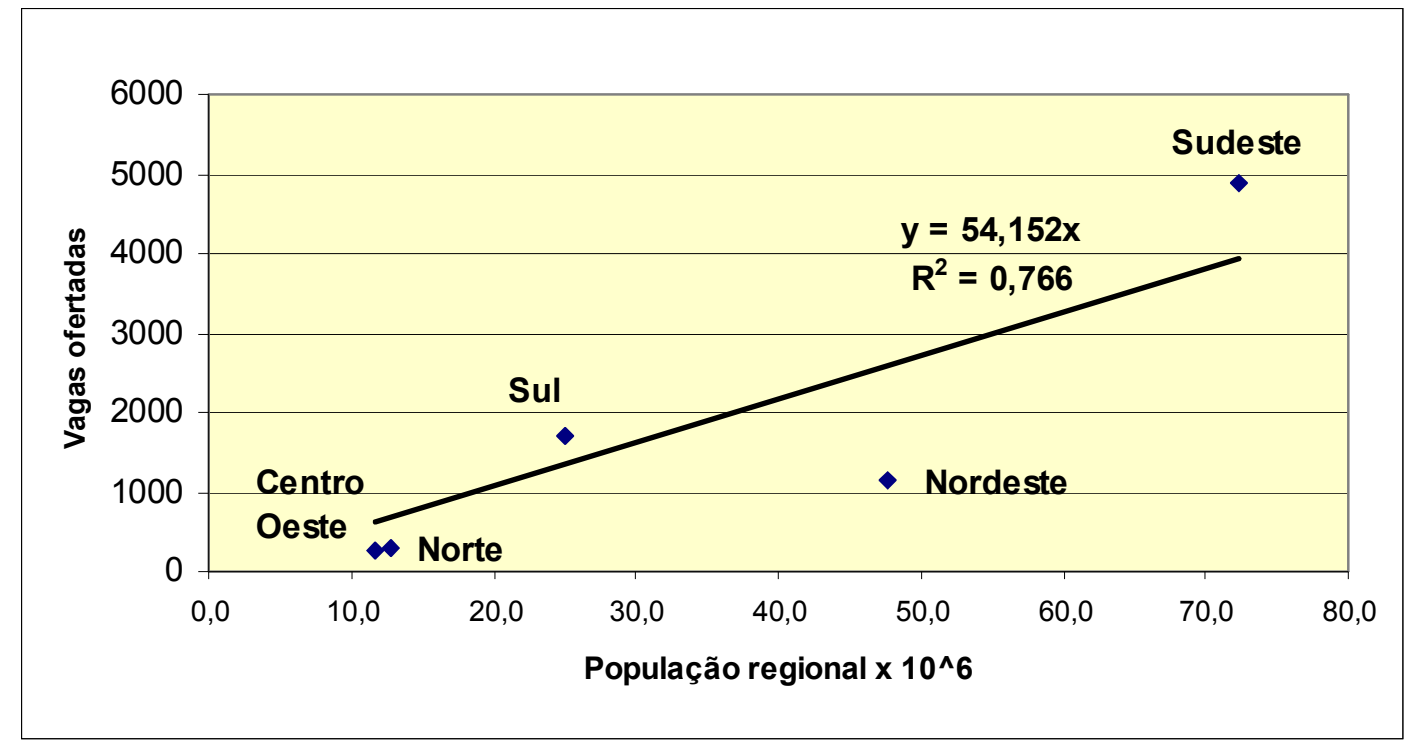

Figura 5 - Oferta de vagas em função da população regional

Analisando as figuras 4 e 5, observa-se que a região Nordeste é um ponto que se afasta da reta de ajuste. A região Nordeste, que possui uma população próxima de 50 milhões de habitantes, detém apenas 15 cursos de EP. Se comparado com a região Sul, por exemplo, esta possui uma população 50\% menor, contudo, mantém 27 cursos de EP, um número bem superior. Esses números podem ser explicados, parcialmente, por questões sócio-econômicas enfrentadas pelos estados da 
região Nordeste. No entanto, o tamanho da população, e conseqüente mão de obra disponível, exigiriam Engenheiros de Produção para organizar o trabalho e estruturar sistemas produtivos. Esses dados indicam que a oferta de cursos de EP no Nordeste é insuficiente.

As retas de ajuste, que aparecem nas figuras 3 e 5, permitem fazer outra análise importante, referente aos valores médios nacionais de Vagas de EP/PIB e Vagas de EP/População. A figura 3 revela que, no Brasil, em média, são ofertados 7,7 vagas para cada um millhão de PIB. Assim, para um estado (ou conjunto de municípios) que tivesse um PIB anual de, por exemplo, dez milhões seria esperada uma oferta de 77 vagas/ano em cursos de graduação em EP.

Similarmente, a figura 5 revela que, no Brasil, em média, são ofertados 54,2 vagas para cada um millhão de pessoas. Assim, para um estado (ou conjunto de municípios) que tivesse uma população de, por exemplo, dois milhões de habitantes seria esperada uma oferta de 108 vagas/ano em cursos de graduação em EP.

Naturalmente, essas são indicações médias, que servem como um primeiro ponto de referência. A demanda efetiva é influenciada por muitas variáveis, entre elas, o perfil de industrialização da região. Mais ainda, tendo em vista a grande procura pelos cursos de graduação em Engenharia de Produção, caracterizada por demandas no concurso vestibular da ordem de 5 ou mais candidatos por vaga, os números apresentados poderiam ser considerados limites mínimos para a oferta de vagas.

Enquanto proposta para trabalhos futuros, seria interessante confrontar os números observados no Brasil com dados de alguns países desenvolvidos. Essa comparação poderia fornecer subsídios importantes para definir referenciais para a EP nacional.

\section{Considerações finais}

Este trabalho apresentou um estudo da evolução da EP no Brasil. Esse estudo contemplou o cruzamento da oferta de cursos e vagas na graduação de EP com indicadores econômicos e sociais. A análise da relação entre os cursos e vagas de EP ofertados nas diferentes regiões do Brasil e os correspondentes valores de PIB indicou uma provável insuficiência de oferta na região CentroOeste. A análise da relação entre os cursos e vagas de EP ofertados nas diferentes regiões do Brasil e as correspondentes populações, por sua vez, indicou uma provável insuficiência de oferta na região Nordeste.

Traduzido em números médios, o estudo revelou que, atualmente, no Brasil, são ofertadas 7,7 vagas para cada milhão de PIB, ou, em termos populacionais, são ofertadas 54,2 vagas para cada milhão de habitantes.

Podem ser citadas como limitações e dificuldades no desenvolvimento deste trabalho, a pouca disponibilidade de informações relativas à história da Engenharia de Produção no Brasil e à 
atual situação dos cursos. São poucos os dados e relatos das criações, alterações e andamento dos cursos existentes. Nesse sentido, o estudo configura-se como um importante material de consulta à comunidade acadêmica da Engenharia de Produção no país.

A grande capacidade do Engenheiro de Produção em integrar as questões técnicas com as gerenciais tem tornado esse profissional muito procurado pelo mercado de trabalho. Sabe-se que grande parte dos problemas enfrentados no dia-a-dia das empresas envolve questões gerenciais, exigindo domínio das áreas técnica e administrativa. É nesse contexto que o Engenheiro de Produção exerce forte atuação e, sobretudo, possui a capacidade de estabelecer a integração necessária entre os diferentes setores das companhias.

\begin{abstract}
In the last decade, Industrial Engineering has demonstrated a prominent growth in Brazil. This paper presents a quantitative study of the evolution and localization of Brazilian under-graduate courses on Industrial Engineering. Aspects of the evolution of the offer as well as of the number of education institutions that offer the course had been taken in account. The relation of offer with economic and social factors of the regions where these courses are installed was also considered. The results of this study disclose that the offer of courses in Center-West and Northeast regions is relatively small, in view of, respectively, the GIP and population of these regions.
\end{abstract}

Key words: Industrial Engineering, education institutions, under-graduate courses

\title{
Referências
}

ASSOCIAÇÃO BRASILEIRA DE ENGENHARIA DE PRODUÇÃO. Cadastro nacional de cursos. Santa Bárbara do Oeste: ABEPRO, 2003.

COSTA, J. E. Sucesso na Profissão. Revista Veja, n. 1805, p. 76, jun. 2003.

CUNHA, G. D. Um panorama atual da Engenharia da Produção no Brasil. Porto Alegre: [s.n.], 2002.

INSTITUTO BRASILEIRO DE GEOGRAFIA E ESTATÍSTICA. Sistema de Contas Nacionais Brasil 2000. Brasília, DF.: IBGE, 2000.

INSTITUTO BRASILEIRO DE GEOGRAFIA E ESTATÍSTICA. Censo Demográfico 2000. Brasília, DF.: IBGE, 2000.

LEME, R. A. S. A história da Engenharia de Produção no Brasil. In: Encontro Nacional de Engenharia de Produção, 3., São Paulo. Anais... São Paulo, 1983.

MASSOTE, A. A. A Engenharia de Produção no Brasil: evolução e desenvolvimento. Revista Pesquisa e Tecnologia FEI, São Bernardo do Campo, 2002. 